\title{
Nonperturbative Renormalization and the QCD Vacuum
}

\author{
Adam P. Szczepaniak ${ }^{1}$ and Eric S. Swanson ${ }^{2}$ \\ 1 Physics Department and Nuclear Theory Center \\ Indiana University, Bloomington, Indiana 47405-4202 \\ ${ }^{2}$ Department of Physics and Astronomy, University of Pittsburgh, Pittsburgh PA 15260 and \\ Jefferson Lab, 12000 Jefferson Ave, Newport News, VA 23606.
}

\begin{abstract}
We present a self consistent approach to Coulomb gauge Hamiltonian QCD which allows one to relate single gluon spectral properties to the long range behavior of the confining interaction. Nonperturbative renormalization is discussed. The numerical results are in good agreement with phenomenological and lattice forms of the static potential.
\end{abstract}

Typeset using REVTEX 


\section{INTRODUCTION}

There is a long history [1 4] of using mean field techniques to explore dynamical chiral symmetry breaking in Quantum Chromodynamics. Chiral symmetry breaking is of fundamental importance because it is directly related to the vacuum structure of QCD. It also has important practical consequences, such as making chiral perturbation theory a viable approximation to low energy QCD. Mean field equations are typically derived by analyzing a truncation of the Schwinger-Dyson equations or, when dealing with a Hamiltonian, by performing a Bogoliubov-Valatin transformation on the particle basis.

An added complexity with Hamiltonian approaches has to do with renormalization. Unlike covariant approaches to field theory which generate a finite number of counterterms, the Hamiltonian formalism necessarily involves a noncovariant truncation of the theory and hence may generate noncanonical counterterms. As a result it may not be possible to remove the ultraviolet cutoff. In this sense, an effective field theory approach to Hamiltonian-based renormalization becomes natural.

The problem of renormalization in models derived from a QCD Hamiltonian is often ignored. An exception was the work of Adler and Davis [1] who have noted that, when using the BCS Ansatz, consistency with the Ward identities imposes a definite counterterm structure on the gap equation. They also noted that calculations should be performed with the full Hamiltonian (as opposed to the normal ordered Hamiltonian).

Although the quark sector of the vacuum has been studied relatively well, the gluonic sector has largely been ignored. However, the gluonic vacuum is also relevant to studies of chiral symmetry breaking because the quark and gluonic gap equations are coupled and, more importantly, the gluon propagator is related to the quark-antiquark confining interaction. Furthermore, the gluonic vacuum determines the properties of the gluonic quasiparticles which in turn may be used to construct a Fock space expansion of hadrons such as glueballs or hybrids [5].

In this paper we derive and renormalize the gap equation for the purely gluonic sector 
of QCD. This work extends three previous papers. The first of these presented the unrenormalized gluonic gap equation and calculated the gluon condensate and glueball spectrum [5]. The other two [6] described how to renormalize Hamiltonian QCD using the similarity or flow equation methods for evolving the renormalization scale. Both methods are necessarily perturbative, which means that gap equations may be derived with one (or more) loop corrections. However, since the phenomena being studied are nonperturbative, it is preferable to construct a nonperturbative renormalization scheme from the start. Unfortunately, this means abandoning the elegant flow equation methodology which is so well suited to Hamiltonian-based perturbation theory.

We further extend the previous work by showing how to obtain the long range interaction between color sources. This is done in the BCS Ansatz by simultaneously considering the single gluon spectral function and the non-Abelian Coulomb potential. This subject was previously considered in Ref. [8 and [9] (we discuss the various approaches below). We derive a self consistent gap equation for the gluon spectral function. Self consistency arises because the non-Abelian potential appearing in this equation depends on the gluon spectral function. The solution to the gap equation gives rise to an infrared enhancement in the effective non-Abelian potential which may be identified with linear confinement.

In the following section we give a brief review of QCD in Coulomb gauge and present our regularization scheme. We then discuss the renormalization scheme adopted, the gap equation, and the effective interaction emerging from the self consistent solution. We conclude and discuss future applications in Section III.

\section{QCD IN COULOMB GAUGE}

Understanding the properties of soft gluons is one of the major challenges of hadronic physics. It is natural to study soft gluons using the Hamiltonian formulation of QCD in a physical gauge such as the Coulomb gauge. This has many advantages: it is closest in spirit to quantum mechanical models of $\mathrm{QCD}$, all degrees of freedom are physical, no additional 
constraints are need on the Fock space, the norm is positive definite, spurious retardation effects are minimized, and confinement may be rigorously identified with the non-Abelian Coulomb interaction in the heavy quark limit [10]. Finally this is also the natural choice for the study of nonrelativistic bound states of a few constituent degrees of freedom. It is of relevance in the gluonic sector since one would expect the gluonic quasiparticles to be heavy due to strong confining interactions.

The pure gauge QCD Hamiltonian in Coulomb gauge may be written as 11 H $H_{g}+H_{C}$, where the terms are given by,

$$
\begin{aligned}
& H_{g}=\operatorname{Tr} \int d^{3} x\left[\mathcal{J}^{-1} \boldsymbol{\Pi} \cdot \mathcal{J} \mathbf{\Pi}+\mathbf{B} \cdot \mathbf{B}\right] \\
& H_{C}=\frac{1}{2} g^{2} \int d^{3} x d^{3} y \mathcal{J}^{-1} \rho^{a}(\mathbf{x}) K^{a b}(\mathbf{x}, \mathbf{y}) \mathcal{J} \rho^{b}(\mathbf{y})
\end{aligned}
$$

The second term is the instantaneous non-Abelian Coulomb interaction for QCD. The kernel $K$ is given by

$$
K^{a b}(\mathbf{x}, \mathbf{y})=\left\langle\mathbf{x}, a\left|(\nabla \cdot \mathbf{D})^{-1}\left(-\nabla^{2}\right)(\nabla \cdot \mathbf{D})^{-1}\right| \mathbf{y}, b\right\rangle
$$

and the color charge density $\rho^{a}$ is given by

$$
\rho^{a}(\mathbf{x})=f^{a b c} \mathbf{A}^{b}(\mathbf{x}) \cdot \mathbf{\Pi}^{c}(\mathbf{x})
$$

When expanded in powers of the strong coupling, the Coulomb kernel contains infinitely many terms arising from the inverse of the adjoint covariant derivative:

$$
\mathbf{D}^{a b}=\delta^{a b} \nabla-g f^{a b c} \mathbf{A}^{c} \cdot \nabla
$$

At lowest order in the coupling the kernel is given by $K^{a b}(\mathbf{x}, \mathbf{y})=\delta^{a b} /(4 \pi|\mathbf{x}-\mathbf{y}|)$ as in QED. The Faddeev-Popov determinant appears in the kinetic energy and Coulomb interaction terms due to the curvature of the gauge manifold and is given by $\mathcal{J}=\operatorname{det}[\nabla \cdot \mathbf{D}]$. Lastly, the components of the non-Abelian magnetic field are given by

$$
B_{i}^{a}=\epsilon_{i j k}\left(\nabla_{j} A_{k}^{a}+\frac{g}{2} f^{a b c} A_{j}^{b} A_{k}^{c}\right)
$$


It should be stressed that this is a bare Hamiltonian. Regularization leads to counterterms which in practice will depend on the approximation schemes used to diagonalize the Hamiltonian. As stated above, our goal is to study the QCD vacuum with the aid of the BCS Ansatz. In the past we have chosen to regulate the Hamiltonian by restricting its matrix elements in the basis of eigenstates of the bare kinetic energy to a band-diagonal form [6,7]. Although an elegant formulation of the renormalization procedure is possible with this choice of basis, it gives rise to lengthy expressions because all calculations must be performed in the particle basis. Furthermore, such a scheme does not seem to be particularly relevant when dealing with nonperturbative renormalization since there is no particular advantage in using the bare basis. Hence, it is preferable to employ the field basis and we introduce a field-based regulator which is analogous to Schwinger's point splitting. This consists of smearing field operators over a small spatial region:

$$
\tilde{\mathrm{A}}_{i}^{b}(\mathbf{x})=\int d^{3} y \frac{\Lambda^{3}}{(2 \pi)^{3 / 2}} \mathrm{~A}_{i}^{b}(\mathbf{y}) \mathrm{e}^{-(\mathbf{x}-\mathbf{y})^{2} \frac{\Lambda^{2}}{2}}
$$

Here $\Lambda$ is the UV cutoff and the fields are effectively smeared over a distance $O(1 / \Lambda)$. The mode expansions are

$$
\begin{aligned}
& \tilde{\mathrm{A}}_{i}^{b}(\mathbf{x})=\int \frac{d^{3} k}{(2 \pi)^{3}} \frac{1}{\sqrt{2 \omega_{0}(k)}}\left(a_{i}^{b}(\mathbf{k})+a_{i}^{b \dagger}(-\mathbf{k})\right) \mathrm{e}^{-i \mathbf{k} \cdot \mathbf{x}-\frac{k^{2}}{2 \Lambda^{2}}} \\
& \tilde{\Pi}_{i}^{b}(\mathbf{x})=\int \frac{d^{3} k}{(2 \pi)^{3}} i \sqrt{\frac{\omega_{0}(k)}{2}}\left(a_{i}^{b}(\mathbf{k})-a_{i}^{b \dagger}(-\mathbf{k})\right) \mathrm{e}^{-i \mathbf{k} \cdot \mathbf{x}-\frac{k^{2}}{2 \Lambda^{2}}}
\end{aligned}
$$

where $\omega_{0}(k)=|\mathbf{k}|$ in the perturbative vacuum. We shall subsequently drop the tildes. Contractions of the field operators which are needed below are given as follows

$$
\begin{aligned}
\left\langle\mathrm{A}_{i}^{a}(\mathbf{x}) \mathrm{A}_{j}^{b}(\mathbf{y})\right\rangle & =\delta^{a b} \int \frac{d^{3} k}{(2 \pi)^{3}} \frac{D_{i j}(k)}{2 \omega_{0}(k)} \mathrm{e}^{i \mathbf{k} \cdot(\mathbf{x}-\mathbf{y})-\frac{k^{2}}{\Lambda^{2}}} \\
\left\langle\Pi_{i}^{a}(\mathbf{x}) \Pi_{j}^{b}(\mathbf{y})\right\rangle & =\delta^{a b} \int \frac{d^{3} k}{(2 \pi)^{3}} D_{i j}(k) \frac{\omega_{0}(k)}{2} \mathrm{e}^{i \mathbf{k} \cdot(\mathbf{x}-\mathbf{y})-\frac{k^{2}}{\Lambda^{2}}} \\
\left\langle\mathrm{~A}_{i}^{a}(\mathbf{x}) \Pi_{j}^{b}(\mathbf{y})\right\rangle & =i \delta^{a b} \int \frac{d^{3} k}{(2 \pi)^{3}} \frac{D_{i j}(k)}{2} \mathrm{e}^{i \mathbf{k} \cdot(\mathbf{x}-\mathbf{y})-\frac{k^{2}}{\Lambda^{2}}}
\end{aligned}
$$

where $D_{i j}(k)=\delta_{i j}-\hat{k}_{i} \hat{k}_{j}$ is the transverse delta function associated with Coulomb gauge. 


\section{A. Nonperturbative Renormalization}

In order to reduce the cutoff dependence induced by regularization we allow the couplings to be $\Lambda$-dependent and add an infinite set of counterterms to the Hamiltonian. The counterterms are organized in powers of the cutoff,

$$
\delta H \equiv \sum_{n=-2} \frac{c_{n}(\Lambda)}{\Lambda^{n}} \mathcal{O}^{(n)} .
$$

As long at the cutoff only affects the operator products at short relative distances, the counterterms $\mathcal{O}$ must be local. Thus they may be classified according to their canonical dimension, $n+1$. Furthermore they have to preserve the unbroken symmetries i.e. be rotationally invariant, color, spin, and flavor singlets. Notice that the series starts at order $\Lambda^{2}$. This is because the lowest dimension operator satisfying the above restrictions is $\int d \mathbf{x A}^{2}(\mathbf{x})$ which is of dimension $-1,(n=-2)$. The needed marginal and relevant operators contributing to the gluon sector of the Hamiltonian are

$$
\begin{aligned}
\delta H= & \frac{M^{2}(\Lambda)}{2} \int d^{3} x A^{2}+\frac{c_{0}(\Lambda)}{2} \int d^{3} x A \nabla^{2} A \\
& +\frac{Z^{-1}(\Lambda)-1}{2} \int d^{3} x \Pi^{2}+\frac{Z(\Lambda)-1}{2} \int d^{3} x \mathbf{B}^{2}
\end{aligned}
$$

We have established contact with more traditional notation by defining $M^{2}(\Lambda)=\Lambda^{2} c_{-2}$ where $c_{-2}$ is dimensionless. Operators of dimension three and five do not occur. Thus the next set of relevant operators are of dimension six: $f_{a b c} f_{c d e} A^{i a} \Pi^{i b} A^{j d} \Pi^{j e}, A \cdot \Pi A \cdot \Pi, A \nabla^{4} A$, $\Pi \nabla^{2} \Pi, f_{a b c} \Pi^{i a} A^{b} \cdot \nabla \Pi^{i c}$, combinations of six gluon fields and gradients, and so on.

It is important to recall that in an effective field theory the cutoff is not removed. Rather the various coefficients are determined by requiring that observables are accurate to a given order in $p / \Lambda$, where $p$ is some characteristic, measurable momentum scale. In our case the $c_{n}(\Lambda)$ should be tuned to reproduce experimental data calculated in some nonperturbative scheme. Here we are referring to a number of standard many-body calculational schemes such as the BCS vacuum Ansatz, the Tamm-Dancoff truncation, or the random phase approximation. 
Transverse gluons do not contribute to the counterterms because we employ a BCS Ansatz in this work. Thus at this stage of the calculation, the $\Lambda$ dependence of the counterterms will not match that of perturbative QCD. Nevertheless couplings to the transverse

gluons with momenta below the cutoff are still present in the Hamiltonian and lead to important effects which (if the valence sector dominates) could be taken into account in perturbation theory around quasiparticle bound states. Removal of the cutoff without taking into account the effects from transverse gluons would clearly be incorrect. This will be seen explicitly below when we compare, in the weak coupling limit, the counterterms calculated with the BCS Ansatz with the results coming from perturbation theory.

\section{B. The Gap Equation}

There are many equivalent formulations of the BCS approach. The one appropriate to many-body physics is the Bogoliubov-Valatin (BV) canonical transformation on the particle operators. In our case this may be written as

$$
\begin{array}{r}
a_{i}^{b}(\mathbf{k})=c(k) \alpha_{i}^{b}(\mathbf{k})+s(k) \alpha_{i}^{b \dagger}(-\mathbf{k}) \\
a_{i}^{b \dagger}(\mathbf{k})=c(k) \alpha_{i}^{b \dagger}(\mathbf{k})+s(k) \alpha_{i}^{b}(-\mathbf{k})
\end{array}
$$

where the rotation is parameterized in terms of an unknown gap function, $\omega$, as

$$
\begin{aligned}
& c(k)=\frac{1}{2}\left(\sqrt{\frac{\omega_{0}(k)}{\omega(k)}}+\sqrt{\frac{\omega(k)}{\omega_{0}(k)}}\right) \\
& s(k)=\frac{1}{2}\left(\sqrt{\frac{\omega_{0}(k)}{\omega(k)}}-\sqrt{\frac{\omega(k)}{\omega_{0}(k)}}\right) .
\end{aligned}
$$

Because we have employed a field-based regulator, the effect of the BV transformation is to simply replace $\omega_{0}(k)=k$ with $\omega_{0}=\omega(k)$ in the mode expansions of Eq. (9). Thus all matrix elements evaluated in the BCS vacuum are simply related to those evaluated in the perturbative vacuum with the same replacement.

The gap equation may now be obtained from the one-body portion of the QCD Hamiltonian. Normal ordering with respect to the BCS vacuum yields 


$$
H_{1 b}=\int \frac{d^{3} q}{(2 \pi)^{3}} \mathrm{e}^{-\frac{k^{2}}{\Lambda^{2}}}\left[E(q) \alpha_{i}^{b \dagger}(\mathbf{q}) \alpha_{i}^{b}(\mathbf{q})+G(q)\left(\alpha_{i}^{b}(\mathbf{q}) \alpha_{i}^{b}(-\mathbf{q})+H . c .\right)\right]
$$

where

$$
\begin{aligned}
E(q)= & \frac{1}{2 \omega(q)}\left[\frac{\omega^{2}(q)}{Z}+Z q^{2}+M^{2}(\Lambda)+q^{2} c_{0}(\Lambda)\right. \\
& +\frac{N_{c}}{4} \int \frac{d^{3} k}{(2 \pi)^{3}} \mathrm{e}^{-\frac{k^{2}}{\Lambda^{2}}} \tilde{V}(\mathbf{k}+\mathbf{q})\left(1+(\hat{k} \cdot \hat{q})^{2}\right) \frac{\omega^{2}(k)+\omega^{2}(q)}{\omega(k)} \\
& \left.+\pi \alpha_{s}(\Lambda) N_{c} \int \frac{d^{3} k}{(2 \pi)^{3}} \mathrm{e}^{-\frac{k^{2}}{\Lambda^{2}}} \frac{\left(3-(\hat{k} \cdot \hat{q})^{2}\right)}{\omega(k)}\right]
\end{aligned}
$$

and

$$
\begin{aligned}
G(q) & =\frac{1}{4 \omega(k)}\left[-\frac{\omega^{2}(q)}{Z}+Z q^{2}+M^{2}(\Lambda)+c_{0}(\Lambda) q^{2}\right. \\
& +\frac{N_{c}}{4} \int \frac{d^{3} k}{(2 \pi)^{3}} \tilde{V}(\mathbf{k}+\mathbf{q}) \mathrm{e}^{-\frac{k^{2}}{\Lambda^{2}}}\left(1+(\hat{k} \cdot \hat{q})^{2}\right) \frac{\omega^{2}(k)-\omega^{2}(q)}{\omega(k)} \\
& \left.+\pi \alpha_{s}(\Lambda) N_{c} \int \frac{d^{3} k}{(2 \pi)^{3}} \mathrm{e}^{-\frac{k^{2}}{\Lambda^{2}}} \frac{\left(3-(\hat{k} \cdot \hat{q})^{2}\right)}{\omega(k)}\right]
\end{aligned}
$$

The spectral function $\omega(k)$ is obtained from solving the gap equation

$$
G(q)=0
$$

which demands that the quasiparticle, BCS vacuum decouples from states with pairs of gluons - a feature reminiscent of the constituent quark model.

The static potential in these expressions is the expectation value of the non-Abelian Coulomb kernel in the BCS vacuum state,

$$
\tilde{V}(\mathbf{q})=\int d^{3} r \mathrm{e}^{-i \mathbf{q} \cdot \mathbf{r}}\left\langle B C S\left|K^{a b}(\mathbf{r}, \mathbf{0})\right| B C S\right\rangle
$$

At lowest order in the coupling and in the perturbative vacuum this potential is simply $4 \pi \alpha_{s}(\Lambda) / q^{2}$. It is possible to show that the non-Abelian Coulomb term describes the complete quark-antiquark interaction in the heavy quark limit [10]. Thus one may use lattice data to determine that the potential takes on the familiar Coulomb+linear form. It is natural to assume that, with the appropriate color structure, this form holds for gluonic color sources as well (in fact this is confirmed by lattice calculations [15]). Thus, in previous 
calculations [10:7] we have simply replaced the expectation of the non-Abelian kernel with a linear potential. However, one of our current goals is to demonstrate that the BCS Ansatz and linear confinement can be described consistently by the same formalism. The central idea is that the gap equation contains a kernel which is itself the expectation value of the non-Abelian Coulomb interaction in the BCS vacuum. Thus the kernel is functionally dependent on the gap function and it is possible to obtain both the gap function and the effective potential. Since it is known that the non-Abelian Coulomb interaction gives rise to confinement in the heavy quark limit, one can hope that this procedure will yield a linear potential at large distances [8], [9].

In an attempt to gain some insight into this hypothesis, we consider perturbative corrections to the effective potential. As stated above, the non-Abelian Coulomb kernel is an operator which depends on the transverse degrees of freedom of the gauge field (cf. Eq. (3)). It can be shown that the $\beta$ function can be obtained from loop corrections to this kernel [13]. One finds that at $O\left(\alpha_{s}\right)$, there are three contributions to $\beta=-\beta_{0} /(2 \pi) \cdot \alpha_{s}^{2}$ : $\beta_{0}=\beta_{c}+\beta_{g}+\beta_{q}=+4 N_{c}+(-1)+\left(-2 n_{f} / 3\right)$. The first term, $\beta_{c}$ comes directly from the expansion of the non-Abelian Coulomb potential. The second and third terms are due to mixing with two-gluon and two-quark intermediate states respectively. In the following we consider the perturbative corrections to the Coulomb kernel since it gives rise to the majority of the pure gauge $\beta$ function.

The contribution from the transverse gluons in the expansion of the Coulomb kernel may be computed with the expansion

$$
\left[\frac{1}{\boldsymbol{\nabla} \cdot \mathbf{D}}\left(-\boldsymbol{\nabla}^{2}\right) \frac{1}{\boldsymbol{\nabla} \cdot \mathbf{D}}\right]_{a b}=-\frac{1}{\boldsymbol{\nabla}^{2}}\left[\delta_{a b}+2 g_{1} \frac{1}{\boldsymbol{\nabla}^{2}} f_{a b c} \mathbf{A}^{c} \cdot \boldsymbol{\nabla}+3 g_{2} \frac{1}{\nabla^{2}} f_{a c d} \mathbf{A}^{d} \cdot \boldsymbol{\nabla} \frac{1}{\nabla^{2}} f_{c b e} \mathbf{A}^{e} \cdot \boldsymbol{\nabla}+\ldots\right]
$$

with the cutoff dependence of the couplings, $g_{i} \equiv g(\Lambda) Z_{i}(\Lambda)$ to be determined. To order $g_{i}^{2}$, the Coulomb interaction is given by

$$
V_{c}(q)=\frac{4 \pi \alpha_{s}}{q^{2}}\left[1+\frac{3 Z_{2}^{2}(\Lambda) \alpha_{s}(\Lambda) \beta_{c}}{16 \pi^{2}} I\left(q, \Lambda ; \omega_{0}\right)\right]
$$


where $\alpha_{s}(\Lambda)=g^{2}(\Lambda) / 4 \pi$ and

$$
I\left(q, \Lambda ; \omega_{0}\right)=\int d^{3} \mathbf{k} \frac{\left(1-(\hat{\mathbf{k}} \cdot \hat{\mathbf{q}})^{2}\right) \mathrm{e}^{-k^{2} / \Lambda^{2}}}{\omega_{0}(k)(\mathbf{q}-\mathbf{k})^{2}} .
$$

In the BCS vacuum the same expression arises except that $\omega_{0}$ is replaced with the unknown function, $\omega$. Thus, as stated above, the solution to the gap equation depends on a potential which itself is a function of the gluon dispersion relation. It therefore becomes possible to obtain the gluon gap function and the nonperturbative potential in a self consistent fashion.

We pursue this scenario by defining a nonperturbative coupling via the relation

$$
V_{c}(q) \equiv \frac{4 \pi \tilde{\alpha}_{e f f}\left(q^{2}\right)}{q^{2}} .
$$

Furthermore, we take part of the rainbow-ladder approximation to $V_{c}$ by summing all diagrams proportional to $I^{n}$. This leads to an expression familiar from the perturbative leading $\log$ approximation,

$$
\tilde{\alpha}_{e f f}\left(q^{2}\right)=\frac{\alpha_{s}(\Lambda)}{1-\frac{3 Z_{2}^{2}(\Lambda) \alpha_{s}(\Lambda) \beta_{c}}{16 \pi^{2}} I(q, \Lambda ; \omega)} .
$$

Although this potential may be inserted directly into the gap equation we have found it useful to approximate it with the aid of the following substitution

$$
I[\omega] \rightarrow \frac{4 \pi}{3} \log \left[\frac{\Lambda^{2}}{\omega(q)^{2}}\right]
$$

As shown in Fig. 1, this form is accurate to roughly $10 \%$ for $q / \Lambda<0.1$. 


\section{FIGURES}

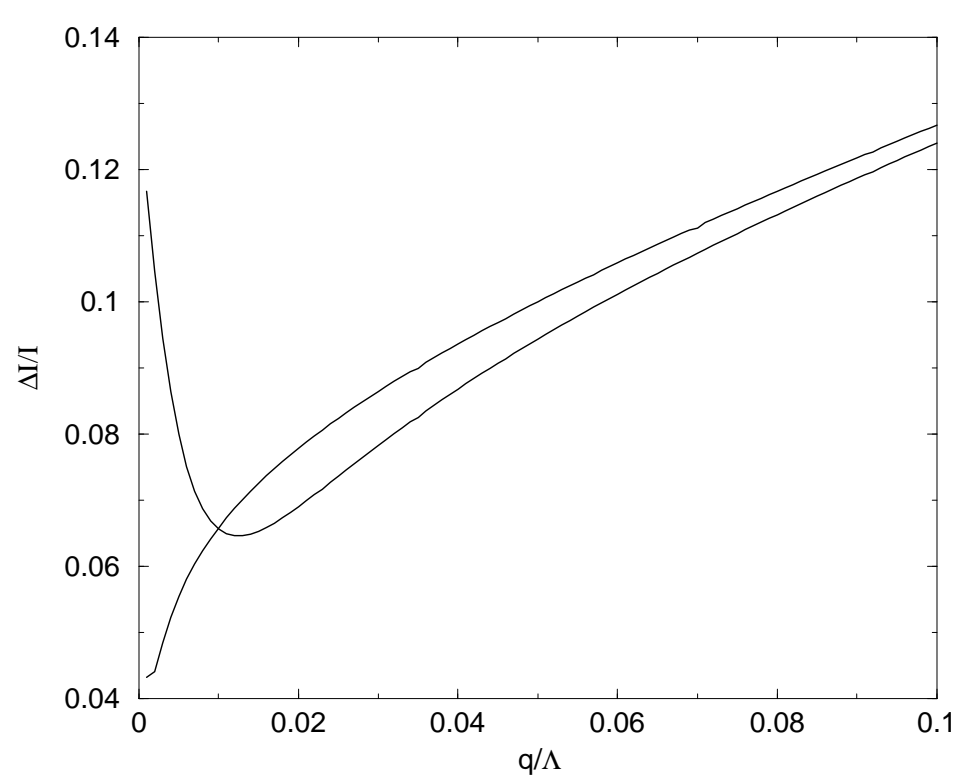

FIG. 1. Relative error due to approximation defined in Eq. (29). The ratio $\left(I[\omega]-\log \left[\frac{\Lambda^{2}}{\omega(q)^{2}}\right]\right) / I[\omega]$ is plotted as a function of $q / \Lambda$ for $\omega(q)^{2} \equiv M^{2}+q^{2}$. The two curves correspond to $M / \Lambda=0.01$ and $M / \Lambda=0.1$ (larger difference for smaller $q$ ).

With this expression for $V_{c}$, the gap equation is given by

$$
\begin{aligned}
\frac{\omega(q)^{2}}{Z} & =Z q^{2}+M^{2}(\Lambda)+c_{0}(\Lambda) q^{2}+\pi \alpha_{s}(\Lambda) N_{c} \int \frac{d^{3} k}{(2 \pi)^{3}} \mathrm{e}^{-\frac{k^{2}}{\Lambda^{2}}} \frac{\left(3-(\hat{k} \cdot \hat{q})^{2}\right)}{\omega(k)} \\
& +\frac{N_{c}}{4} \int \frac{d^{3} k}{(2 \pi)^{3}} \mathrm{e}^{-\frac{k^{2}}{\Lambda^{2}}} \tilde{V}_{c}(\mathbf{k}+\mathbf{q})\left(1+(\hat{q} \cdot \hat{k})^{2}\right) \frac{\omega^{2}(k)-\omega^{2}(q)}{\omega(k)}
\end{aligned}
$$

Many of the terms of higher order in $1 / \Lambda$ also contribute to the gap equation. Additional terms include $c_{2}(\Lambda) q^{2} \omega(q)^{2} / \Lambda^{2}, d_{2}(\Lambda) q^{4} / \Lambda^{2}$, and $e_{2}(\Lambda) / \Lambda^{2} \cdot N_{c} / 3 \cdot \int \exp \left(-k^{2} / \Lambda^{2}\right)\left(\omega(k)^{2}-\right.$ $\left.\omega(q)^{2}\right) / \omega(k)$. As expected, these only affect the solution at short range, thus the long range behavior of the effective potential is completely specified by the nonperturbative model (the BCS Ansatz in this case). Nevertheless, one may get an indication about the viability of the low energy model by studying the coefficients, $c_{2}, d_{2}$, etc - a strong cutoff dependence at low momenta would indicate that the nonperturbative model is inadequate and that additional counterterms are therefore required.

At this point the standard procedure would be to choose values for the cutoff and coeffi- 
cients, solve the gap equation to obtain $\omega$, use the solution to obtain, say, glueball masses[t, and vary the coefficients so that the predictions agree with experimental (or lattice) data. However, in this study we choose to perform a simpler analysis and fit the derived form of the effective potential, $V_{e f f}=\tilde{V}(\omega)$ to the Wilson loop lattice potential. This is similar in spirit to a lattice renormalization procedure advocated by Lepage and Mackenzie [16]. Dimension six operators are neglected so that the couplings to be varied are the five coefficients $Z, c_{-2}, c_{0}, Z_{2}$, and $\alpha_{s}$. First we note that not all of the coefficients are independent since one requires that the effective potential, and therefore $\omega$, is $\Lambda$ independent (to leading order in $p / \Lambda)$. Multiplying the gap equation by $Z$ and introducing $\tilde{\alpha}_{s}=Z \alpha_{s}, \tilde{c}_{-2}=Z\left(c_{-2}+L\right)$, $\tilde{c}_{0}=Z^{2}+Z c_{0}$ leads to

$\omega^{2}(q)=\tilde{c}_{0}(\Lambda) q^{2}+\Lambda^{2} \tilde{c}_{-2}(\Lambda)+\frac{N_{c}}{(4 \pi)^{2}} \int_{0}^{\infty} d k k^{2} d(\hat{q} \cdot \hat{k}) \tilde{V}_{c}(\mathbf{k}+\mathbf{q})\left(1+(\hat{q} \cdot \hat{k})^{2}\right) \frac{\omega^{2}(k)-\omega^{2}(q)}{\omega(k)}$

where

$$
\tilde{V}_{c}(q)=\frac{4 \pi \tilde{\alpha}_{s}(\Lambda)}{q^{2}\left(1-\frac{\tilde{\alpha}_{s}(\Lambda) \beta_{c}}{4 \pi} \log \left[\frac{\Lambda^{2}}{\omega^{2}(q)}\right]\right)}
$$

and $L$ is defined to be the constant, $\Lambda$-dependent contribution from the third term on the right hand side of Eq. (30) (this term comes from the $A^{4}$ operator in the Hamiltonian). In obtaining Eq. (31) we have further chosen $Z=Z_{2}^{2}$ which is required to make the effective interaction cutoff independent.

We now define a QCD scale, $\mu$, as

$$
\mu^{2} \equiv \Lambda^{2} \mathrm{e}^{-\frac{4 \pi}{\alpha_{s}(\Lambda) \beta_{c}}}
$$

and set $m^{2}(q) \equiv \omega^{2}(q)-q^{2}$. The expression for the running coupling becomes cutoff independent (as long as $m(q)$ is $\Lambda$-independent) and is given by

\footnotetext{
${ }^{1}$ This procedure is followed, without renormalization, in Ref. [5].
} 


$$
\alpha_{e f f}\left(q^{2}\right)=\frac{4 \pi}{\beta_{c} \log \left(\frac{q^{2}+m(q)^{2}}{\mu^{2}}\right)}, \quad \tilde{V}_{c}(q)=\frac{4 \pi \alpha_{e f f}\left(q^{2}\right)}{q^{2}} .
$$

We note that if $m(0)=\mu$ the long range behavior of the effective potential corresponds to a linear potential in position space. Equating the coefficient of the leading $1 / q^{4}$ behavior with the standard form $6 \pi b / q^{4}$ ( $b$ is the string tension), yields $\mu^{2}=3\left(1+m^{\prime}\right) \beta_{c} b /(8 \pi)$ where $m^{\prime}=d m^{2}(0) / d q^{2}$. The two remaining coefficients in the gap equations can thus be fixed by demanding that the effective potential represents linear confinement with the right slope. In numerical computations, instead of fixing $m^{\prime}$ we have chosen to fix $m^{2}(q)$ at a finite value of $q^{2}$, typically $q^{2}=1 \mathrm{GeV}^{2}$, which is numerically easier. The two renormalization conditions, one for $m(0)$ and one for $m^{\prime}$ (or in general for $m^{2}\left(q_{0}\right)$ and $m^{2}\left(q_{1}\right)$ ), can be implemented directly into the gap equation by two subtractions,

$$
\begin{aligned}
\omega^{2}(q) & =q^{2} \frac{\omega_{1}^{2}-\omega_{0}^{2}}{q_{1}^{2}-q_{0}^{2}}+\frac{q_{1}^{2} \omega_{0}^{2}-q_{0}^{2} \omega_{1}^{2}}{q_{1}^{2}-q_{0}^{2}}+\int_{0}^{\infty} d k \mathcal{V}(k, q) \frac{\omega^{2}(k)-\omega^{2}(q)}{\omega(k)} \\
& +\frac{q^{2}-q_{1}^{2}}{q_{1}^{2}-q_{0}^{2}} \int_{0}^{\infty} d k \mathcal{V}\left(k, q_{0}\right) \frac{\omega^{2}(k)-\omega_{0}^{2}}{\omega(k)}+\frac{q_{0}^{2}-q^{2}}{q_{1}^{2}-q_{0}^{2}} \int_{0}^{\infty} d k \mathcal{V}\left(k, q_{1}\right) \frac{\omega^{2}(k)-\omega_{1}^{2}}{\omega(k)}
\end{aligned}
$$

where $\omega_{0}=\omega\left(q_{0}\right), \omega_{1}=\omega\left(q_{1}\right)$ and

$$
\mathcal{V}(k, q)=\frac{N_{c}}{(4 \pi)^{2}} \int_{-1}^{1} d(\hat{q} \cdot \hat{k}) k^{2} \mathrm{e}^{-\frac{k^{2}}{\Lambda^{2}}} \tilde{V}_{c}(\mathbf{k}+\mathbf{q})\left(1+(\hat{q} \cdot \hat{k})^{2}\right)
$$

The original couplings may be reconstructed as follows:

$$
\begin{aligned}
& \Lambda^{2} \tilde{c}_{-2}=\frac{q_{1}^{2} \omega_{0}^{2}-q_{0}^{2} \omega_{1}^{2}}{q_{1}^{2}-q_{0}^{2}}-\frac{q_{1}^{2}}{q_{1}^{2}-q_{0}^{2}} \int_{0}^{\infty} d k \mathcal{V}\left(k, q_{0}\right) \frac{\omega^{2}(k)-\omega_{0}^{2}}{\omega(k)}+\frac{q_{0}^{2}}{q_{1}^{2}-q_{0}^{2}} \int_{0}^{\infty} d k \mathcal{V}\left(k, q_{1}\right) \frac{\omega^{2}(k)-\omega_{1}^{2}}{\omega(k)} \\
& \tilde{c}_{0}=\frac{\omega_{1}^{2}-\omega_{0}^{2}}{q_{1}^{2}-q_{0}^{2}}-\frac{1}{q_{1}^{2}-q_{0}^{2}} \int_{0}^{\infty} d k \mathcal{V}\left(k, q_{1}\right) \frac{\omega^{2}(k)-\omega_{1}^{2}}{\omega(k)}+\frac{1}{q_{1}^{2}-q_{0}^{2}} \int_{0}^{\infty} d k \mathcal{V}\left(k, q_{0}\right) \frac{\omega^{2}(k)-\omega_{0}^{2}}{\omega(k)}(38)
\end{aligned}
$$

The single gluon energy is given by

$$
E(q)=\omega(q)\left[Z^{-1}+\int_{0}^{\infty} d k \frac{\mathcal{V}(k, q)}{\omega(k)}\right]
$$

If a linear potential is to be recovered (as it must because we are fitting to lattice data), one must have $\omega_{0}=\mu$ at $q_{0}=0$, (see Eq. (34)). Thus the QCD scale $\mu$ must be related to the 
string tension. As discussed above, instead of taking the limit $q_{1} \rightarrow q_{0}$ and fixing $d \omega^{2}(0) / d q^{2}$ to reproduce the magnitude of string tension we keep $q_{1}$ finite, choose $q_{1}=1 \mathrm{GeV}$ and fit $\omega_{1}$ to reproduce the strength of the confining potential at low momentum. In our computations the one dimensional nonlinear integral (after numerical evaluation of the angular integrals) equation was solved numerically using a modified Levenberg-Marquardt algorithm. We note that the equations are extremely sensitive to the proper treatment of the IR singularity (which, however, is integrable) and that some solutions which have appeared in the literature are misleading. The results for $\alpha_{e f f}(q)=q^{2} V_{e f f}(q) / 4 \pi$ are shown in Fig. 2. We find that $m^{\prime} \sim 0$ (typically $m^{\prime}=0.01$ ) thus the QCD scale $\mu$ can be directly calculated from the physical string tension. Taking $b=0.18 \mathrm{GeV}^{2}$ yields $\mu^{2}=0.2579 \mathrm{GeV}^{2}$. Finally, for large momenta the running of the effective coupling is given by

$$
\alpha_{e f f}^{U V}(q)=\frac{4 \pi}{\beta_{c} \log \left(\frac{q^{2}}{0.2579 G e V^{2}}\right)}
$$

Over the a range of momenta the full solution to the effective coupling (or potential) can be well approximated by a running coupling version of the Cornell potential

$$
\alpha_{e f f}(q)=\frac{4 \pi}{\beta_{c} \log \left[\frac{q^{2}}{\mu^{2}}+\left(\frac{\mu_{0}}{\mu}\right)^{2}\right]}+\frac{3}{2} \frac{b}{q^{2}}=\frac{\alpha_{e f f}^{U V}(\Lambda)}{\left(1+\frac{\alpha_{e f f}^{U V}(\Lambda) \beta_{c}}{4 \pi} \log \frac{p^{2}+\mu_{0}^{2}}{\Lambda^{2}}\right)}+\frac{3}{2} \frac{b}{q^{2}} .
$$

Here $\mu_{0}$ is a parameter which allows interpolation between the infrared and ultraviolet limits of the potential. We find $\left(\mu_{0} / \mu\right)^{2} \sim 10$. Essentially identical results are obtained upon comparison with the standard Coulomb+linear form of the Cornell potential. 


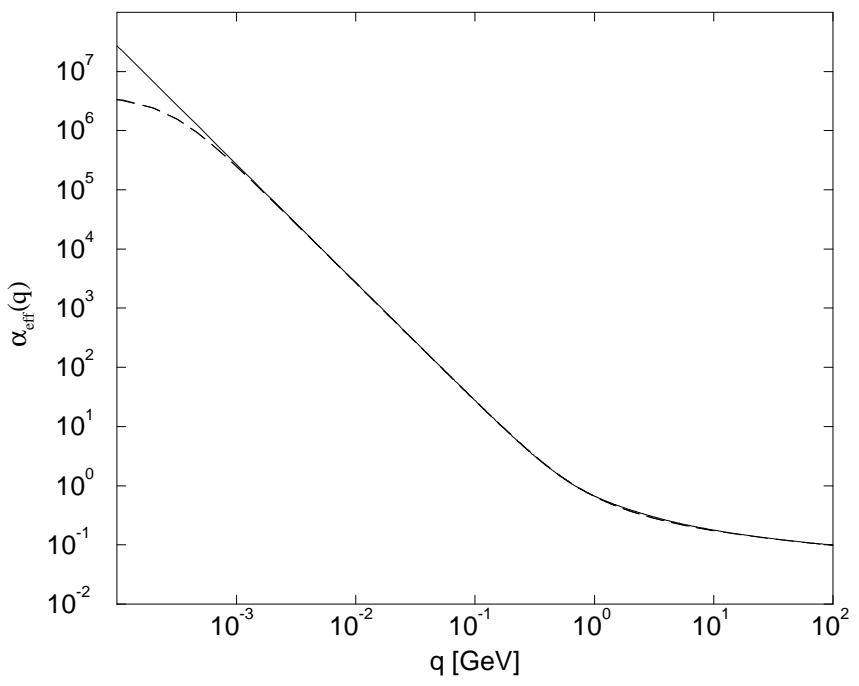

FIG. 2. The effective potential derived from the self-consistent gap equation. The dashed line is the numerical solution to the gap equation with $\Lambda=10 \mathrm{GeV}$ ( $\Lambda$ dependence is negligible and would not be visible on this scale). The solid line is the fit to the running Cornell potential as discussed in the text. The discrepancy at low momenta is due to the finite mesh size in the numerical calculations.

That the form of the derived effective potential so nearly reproduces linear confinement at long range is a tantalizing indication that our goal of deriving the confinement potential in a self-consistent manner from the BCS vacuum Ansatz may be achieved.

The derived gluon dispersion relation $\omega(q)$ is shown in Fig. 3. The doubly subtracted form of the gap equation removes $\Lambda^{2}$ and $\log (\Lambda)$ dependence from the gap equation and leaves subleading terms of order $q^{2} / \Lambda^{2}$. This is reflected in the cutoff independence evident in Fig. 3 at low momentum. In Fig. 3 we also compare the full solution of the gap equation for $\Lambda=10$ with the approximate one obtained using the "Coulomb+linear" potential with the parameters fitted to the self consistent solution as discussed above. Both of them lead to $m^{\prime} \sim 0$ and both are renormalized at the same point, $q_{1}=1 \mathrm{GeV}$, however the intermediate $q$ dependence is somewhat different. As seen from Fig. 2, this small difference does not alter the behavior of the effective interaction. 


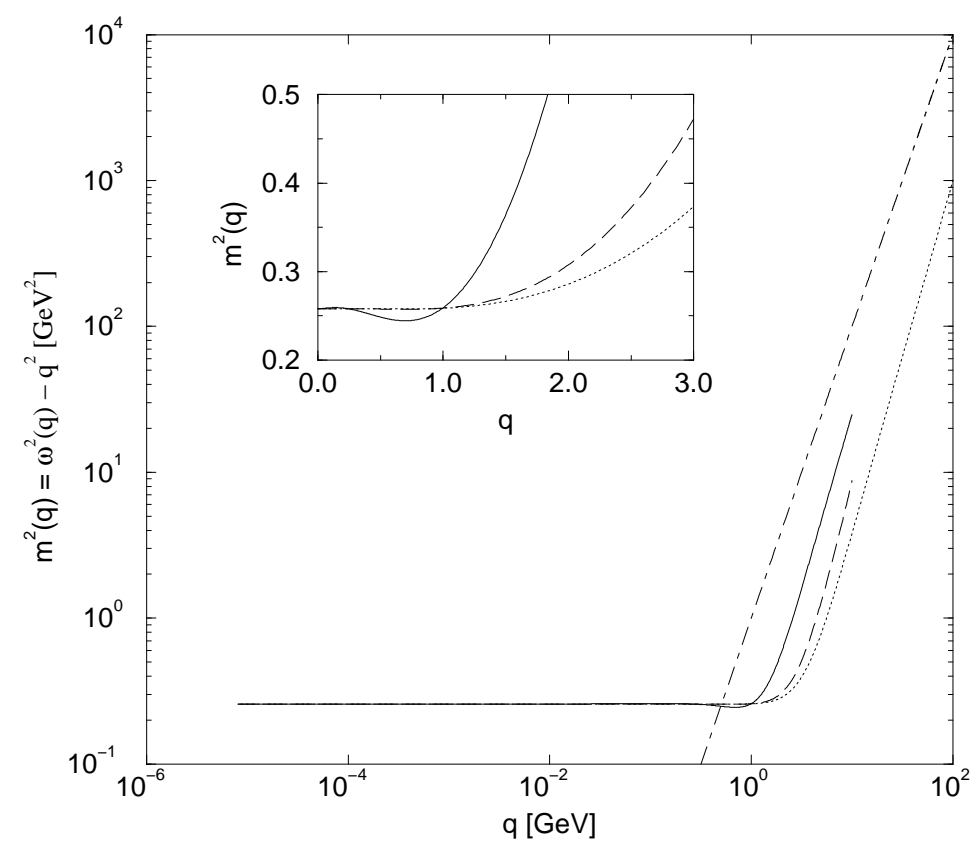

FIG. 3. $\quad m^{2}(q) \equiv \omega^{2}(q)-q^{2}$ is a function of momentum. The dashed and dotted lines correspond to $\Lambda=10 \mathrm{GeV}$ and $\Lambda=100 \mathrm{GeV}$ respectively. The solid line is the Cornell potential fit to the full solution with $\Lambda=10 \mathrm{GeV}$. The dash-dotted line is $m^{2}(q)=q^{2}$.

One may expect that $\omega(q)$ should approach the free solution, $\omega_{0}(q)=q$ at large momenta. However, this need not be true, both in perturbation theory, where logarithms can ruin the asymptotic behavior, and nonperturbatively. This is because the counterterms $Z B^{2}$ and $c_{0} A \nabla A$ give rise to the term $\tilde{c}_{0} q^{2}$ in the gap equation. Renormalizing $\omega(q)$ at a low momentum scales $q_{0}, q_{1}<<\Lambda$ will result in a logarithmic dependence on $\Lambda$ in $\tilde{c}_{0}$. Thus the effective gluon mass $m^{2}(q)=\omega^{2}(q)-q^{2}$ will remain proportional to $q^{2}$ at large momenta.

As mentioned above, this behavior is also true in perturbation theory. In the following we examine the small coupling limit of the counterterm coefficients to establish contact with perturbative QCD. Evaluating Eqns 37 and 38 to $O\left(\alpha_{s}=\tilde{\alpha}_{s}(\Lambda)\right)$ yields

$$
\tilde{c}_{-2}=\frac{\alpha_{s} N_{C}}{4 \pi}\left[-\frac{4}{3}-\frac{8}{3}\right]=-\frac{\alpha_{s} \beta_{0}}{N_{C}}
$$

where the first contribution comes from the vacuum expectation value of the Coulomb potential and the second from the $A^{4}$ term in the Hamiltonian, and 


$$
\tilde{c}_{0}=1+\frac{\alpha_{s} N_{c}}{4 \pi} \frac{8}{15} \frac{q_{1}^{2}+q_{0}^{2}}{q_{1}^{2}-q_{0}^{2}} \ln \left(\frac{q_{0}}{q_{1}}\right)+\frac{\alpha_{s} N_{c}}{4 \pi} \frac{8}{15} \ln \left(\frac{\Lambda^{2}}{q_{0} q_{1}}\right)=\frac{\alpha_{s} N_{c}}{4 \pi} \frac{8}{15} \ln \Lambda^{2}+\text { finite. }
$$

In perturbation theory, to $O\left(\alpha_{s}\right), \Lambda$-dependence of $Z$ and $c_{0}$ follows from Eq. (43) and the requirement that the gluon energy (an observable in perturbation theory) is independent of $\Lambda$. Thus from Eq. (39) it follows that

$$
E(q)=q\left[Z^{-1}+\frac{\alpha_{s} N_{c}}{4 \pi} \frac{4}{3} \ln \frac{\Lambda^{2}}{q^{2}}\right]
$$

which leads to

$$
Z=\frac{\alpha_{s} N_{c}}{4 \pi} \frac{4}{3} \ln \Lambda^{2}+\text { finite }
$$

and finally

$$
c_{0}=-\frac{\alpha_{s} N_{c}}{4 \pi} \frac{32}{15} \ln \Lambda^{2}
$$

These expressions agree with the corresponding expressions in a full perturbative Hamiltonian QCD calculation [7]. Note, however, that in Ref. [7] it has been shown that $c_{0}=0$ to $O\left(\alpha_{s}\right)$. This arises because the self energy contribution from a gluon loop with transverse gluon exchange cancels the contribution from the Coulomb and the $A^{4}$ vacuum expectation values? The BCS calculation only contains the latter two terms. In a nonperturbative calculation based on the BCS Ansatz transverse gluons are confined and the single gluon energy of Eq. (39) becomes IR singular. As a result, transverse gluons only affect observables in bound state calculations of color singlet states.

In Fig. 4 we show the behavior of the counterterms, $\tilde{c}_{-2}$ and $\tilde{c}_{0}$ for small couplings. The points represent the full expressions obtained from Eqns 37 and 38. The lines are the perturbative results given above with the finite portions fit to the full results. As expected the agreement is very good for small coupling. Fig. 4a shows a dramatic dependence of $-\tilde{c_{-2}}$ on the value of the UV cutoff. This dependence originates with the subleading $1 / q^{4}$

\footnotetext{
${ }^{2}$ Note that the UV cutoff $\Lambda$ used in Ref. [7] is by a factor of $\sqrt{2}$ larger then the one used here.
} 
behavior of the effective potential which makes a $\log (\Lambda)$ contribution to $\tilde{c}_{-2}$. The solid lines in Fig. 4a incorporate this behavior. The logarithmic term dominates in the small coupling limit and leads to the large relative splitting seen in the figure.
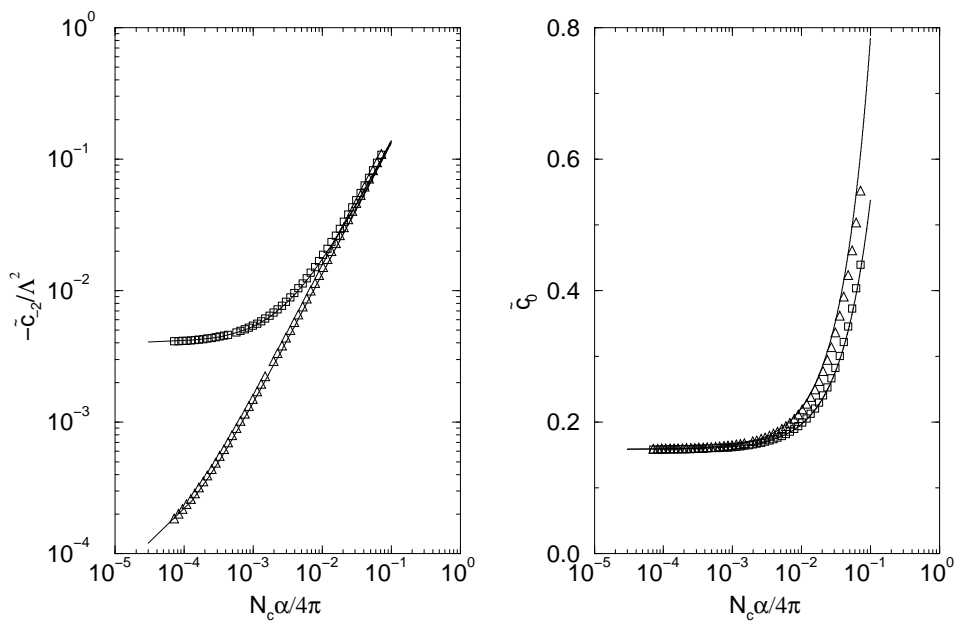

FIG. 4. Counterterms as a function $\alpha_{s}$ in the weak coupling limit. The two set of points correspond to $\Lambda=10 \mathrm{GeV}$ (squares) and $\Lambda=100 \mathrm{GeV}$ (triangles). Solid lines are the results of the leading order $O\left(\alpha_{s}\right)$ perturbative approximation given by Eqs. (42) and (43).

\section{Relationship to Other Approaches}

The Schwinger-Dyson formalism if often used to discuss the issues presented here [14,19]. Advantages are that it is covariant and that calculations beyond the rainbow approximation are possible. However, the resulting integral equations are four dimensional and are therefore difficult to solve. They are also normally evaluated in Euclidean space which introduces interpretational difficulties, especially when dealing with confinement. Furthermore, the connection to the parton model is unclear since it is difficult to continue wavefunctions to Minkowski space. In the Hamiltonian framework color nonsinglets decouple from the

physical spectrum as in the Schwinger-Dyson approach, but hadronic wavefunctions are well-defined, and thus a parton picture of high energy QCD is possible.

We also note that mean field formalisms of Coulomb gauge QCD have been extensively studied in Refs. [8] and [9]. The numerical solution presented in Ref. [8] are however doubtful, 
as they do not have the proper large momentum behavior. In Ref. [9] the Coulomb potential was studied with the aid of a model of the gluon dispersion function (the gap equation was not solved).

A calculation with similar goals to those expressed here has recently appeared [17]. This paper employs the flow equation renormalization methodology to derive a gluonic gap equation which is similar in form to Eq. (30). Differences are that convergence factors have been inserted by hand into two of their loop integrals, a phenomenological potential has been used for the interaction kernel, and the perturbative expression for the gluon mass counterterm has been used. Their calculation also includes transverse gluon contributions at one loop, which is incorrect since the low momentum transverse gluon propagator is strongly modified by the confining interaction. As stated above, the implication of this is that any propagator of color nonsinglet states is IR divergent and that transverse gluons should only be incorporates into color singlet bound state calculations. We stress our belief that the interaction can, and should, be derived in a self-consistent fashion and that the use of perturbative renormalization is not a viable approach to nonperturbative problems. A related problem is seen in their Figure 2 which shows $\omega(q)-q$ approaching zero for $q>4$ $\mathrm{GeV}$. This is not correct, $\omega$ will in fact not approach $q$ at large momentum, but some multiple of $q$.

Curtis and Pennington [14 have noted that the gap equation derived in the SchwingerDyson formalism is not multiplicatively renormalizable. This means that it is not possible to exchange the $\Lambda$-dependence of the gap solution with a renormalization point dependence. As stated above, multiplicative renormalizability is not a concern in the effective field theory. An obvious manifestation of the difference is the appearance of noncanonical counterterms like the gluon mass term. In our approach the cutoff has to be kept finite, otherwise neglected contributions, i.e. from transverse gluons, would cause new divergences to appear. Instead we keep $\Lambda$ finite and use higher dimensional local operators to account for short distance contributions from transverse gluons. In this way, the neglected contributions are kept finite (below the cutoff) and can be evaluated by expanding the Fock space used to diagonalize 
the Hamiltonian.

\section{CONCLUSIONS}

We have presented a computational scheme for evaluating vacuum and quasiparticle properties of QCD. The scheme is based on the QCD Hamiltonian in Coulomb gauge, a field-based regularization, and the effective field theory approach to renormalization. The use of the Hamiltonian is advantageous because all degrees of freedom are physical and a transparent picture of confinement emerges - confinement is generated by the instantaneous non-Abelian Coulomb interaction. We found it useful to regulate the theory by field-smearing because this greatly reduced the complexity of the computations. Field-based calculations have forced us to adopt a field-based renormalization procedure and we have found that the effective field theory approach is ideal for our work.

A gap equation was derived from the QCD Hamiltonian using the Bogoliubov-Valatin transformation. The gap equation necessarily involves the Coulomb interaction kernel which should also be evaluated in the BCS vacuum. Thus self-consistent gap and effective instantaneous color source interaction equations may be derived. With the aid of an approximation to the matrix element of the full kernel in the BCS vacuum, we have found that it is possible to obtain an effective potential which agrees well with the (Abelian) Coulomb and linear regimes of the Wilson loop measurement of the heavy source nonperturbative potential. Furthermore, the derived effective potential is nearly cutoff independent, indicating that a good low energy description has been found.

Encouraged by this success, we look forward to repeating the calculation with the exact BCS interaction kernel. We fully expect this calculation to be equally successful. It will also be of interest to examine the consistency of our results with those derived in the quark sector. Since the quark and gluon vacuum sectors couple at one loop, an examination of the coupled gap equations should prove illuminating. Finally the efficacy of the methodology presented here can be tested by calculating glueball and meson spectra. 


\section{ACKNOWLEDGMENTS}

ES acknowledges support from the DOE under grant DE-FG02-96ER40944 and DOE contract DE-AC05-84ER40150 under which the Southeastern Universities Research Association operates the Thomas Jefferson National Accelerator Facility. AS acknowledges support from the DOE under grant DE-FG02-87ER40365. 


\section{REFERENCES}

[1] S. Adler and A. Davis, Nucl. Phys. B244, 469 (1984).

[2] K.D. Kane, Phys. Rev. D10, 1353 (1974).

[3] A. Amer, A. LeYaouanc, L. Oliver, O. Pène, and J.-C. Raynal, Phys. Rev. Lett. 50, 87 (1983); Phys. Rev. D28, 1530 (1983); LeYaouanc, L. Oliver, O. Pène, and J.-C. Raynal Phys. Rev. D29, 1233 (1984).

[4] J.R. Finger and J.E. Mandula, Nucl. Phys. B199, 168 (1982).

[5] A.P. Szczepaniak, E.S. Swanson, C.-R. Ji, and S.R. Cotanch, Phys. Rev. Lett. 76, 2011 (1996).

[6] A.P. Szczepaniak and E.S. Swanson, Phys. Rev. D55, 1578 (1997).

[7] D.G. Robertson, E.S. Swanson, A.P. Szczepaniak, C.R. Ji, and S.R. Cotanch, Phys. Rev. D59, 074019 (1999).

[8] A.R. Swift, Phys. Rev. D38, 668 (1988).

[9] A. Cucchieri and D. Zwanziger, Phys. Rev. Lett. 78, 3814 (1997).

[10] A.P. Szczepaniak and E.S. Swanson, Phys. Rev. D55, 3987 (1997).

[11] N.H. Christ and T.D. Lee, Phys. Rev. D22, 939 (1980).

[12] J. Schwinger, Phys. Rev. 127, 324 (1962); 130, 402 (1963).

[13] F.L. Feinberg, Phys. Rev. D17, 2659 (1978).

[14] D.C. Curtis and M.R. Pennington, Phys. Rev. D42, 4165 (1990); Phys. Rev. D48, 4933 (1993).

[15] G.I. Poulis and H.D. Trottier, Phys. Lett. B400, 358 (1997); S. Deldar, hep-lat/9911008.

[16] G.P. Lepage and P.B. Mackenzie, Phys. Rev. D48, 2250 (1993). 
[17] E. Gubankova, C.-R. Ji, and S. Cotanch, hep-ph/0003289.

[18] For a pedagogical introduction see G.P. Lepage, nucl-th/9706029.

[19] C.D. Roberts and A.G. Williams, Prog. Part. Nucl. Phys. 33, 477 (1994). 